\title{
Focal application of low-dose-rate brachytherapy for prostate cancer: a pilot study
}

\author{
S. Sara Mahdavi, PhD', Ingrid T. Spadinger, PhD², Septimiu E. Salcudean, PhD³, Piotr Kozlowski, PhD4, \\ Silvia D. Chang, MD, FRCPC ${ }^{5}$, Tony Ng, MD, PhD, FRCPC6, Julio Lobo, MSc ${ }^{3}$, Guy Nir, PhD4, Hamid Moradi, MSc 3 , \\ Michael Peacock, MD', W. James Morris, MD, FRCPC \\ 'Department of Radiation Oncology, Vancouver Centre, British Columbia Cancer Agency, ${ }^{2}$ Department of Medical Physics, Vancouver Centre, \\ British Columbia Cancer Agency, ${ }^{3}$ Department of Electrical and Computer Engineering. University of British Columbia, ${ }^{4}$ Department of Urologic \\ Sciences, University of British Columbia, ${ }^{5}$ Department of Radiology, University of British Columbia, 'Department of Pathology and Laboratory \\ Medicine, University of British Columbia, Vancouver, BC, Canada
}

\begin{abstract}
Purpose: To evaluate the feasibility and to report the early outcomes of focal treatment of prostate cancer using lowdose-rate brachytherapy (LDR-PB).

Material and methods: Seventeen patients were screened with multi-parametric magnetic resonance imaging (mpMRI), 14 of whom proceeded to receive trans-perineal template mapping biopsy (TTMB). Focal LDR-PB was performed on five eligible patients using dual air kerma strength treatment plans based on planning target volumes derived from cancer locations and determined by TTMB. Patient follow-up includes prostate specific antigen (PSA) measurements, urinary and sexual function questionnaires, repeated imaging and TTMB at specific intervals post-treatment.

Results: Feasibility of focal LDR-PB was shown and short-term outcomes are promising. While the detection rate of tumors, a majority of which were low grade GS $3+3$, was found to be low on mpMRI (sensitivity of 37.5\%), our results suggest the potential of mpMRI in detecting the presence of higher grade (GS $\geq 3+4)$, and bilateral disease indicating its usefulness as a screening tool for focal LDR-PB.

Conclusions: Low-dose-rate brachytherapy is a favorable ablation option for focal treatment of prostate cancer, requiring minimal modification to the standard (whole gland) LDR-PB treatment, and appears to have a more favorable side effect profile. Further investigation, in the form of a larger study, is needed to assess the methods used and the long-term outcomes of focal LDR-PB.

J Contemp Brachytherapy 2017; 9, 3: 197-208 DOI: https://doi.org/10.5114/jcb.2017.68424
\end{abstract}

Key words: dual source strength planning, focal therapy, low-dose-rate brachytherapy, multi-parametric MRI, prostate cancer.

\section{Purpose}

Conventional approaches for treating prostate cancer $(\mathrm{PCa})$ include radical treatment, mostly in the form of radical prostatectomy (RP) and radiation therapy. In both methods, the entire gland is targeted, which often results in adverse effects on erectile and urinary function $[1,2,3,4,5]$. For the subset of PCa patients with minimal risk disease, active surveillance (AS) is the only accepted alternative to whole-gland treatment. Patients on AS are routinely monitored and conventional radical treatment is recommended if progression is observed. However, there is a lack of evidence-based consensus defining disease progression and the need for timely radical therapy. Furthermore, monitoring with repeated trans-rectal biopsies carries a risk of systemic infection [6,7], and some men may experience psychological stress knowing that they have an untreated cancer, the behavior of which may be unpredictable $[8,9,10]$. The ProtecT [11] randomized controlled trial, which aims to compare the effectiveness and cost-effectiveness of AS, RP, and radiotherapy in men diagnosed with localized PCa with a median 10-year follow-up, showed no difference between treatment arms with respect to prostate cancer specific mortality or death from any cause; there was, however, a lower incidence of disease progression including development of metastatic disease, favoring upfront treatment with either RP or radiotherapy over AS.

Focal therapy has been proposed as an alternative to radical treatment and active surveillance, and is expected to have an expanding role in the management of PCa $[12,13]$. Even though PCa is frequently multi-focal, some 
studies suggest that the clinically significant disease is often limited to an index lesion $[14,15,16,17,18]$. In such cases, it may be logical to treat only the cancerous part of the gland while preserving healthy tissue.

While an attractive strategy, widespread adoption of focal therapy is held back by three important limitations, namely: 1) inaccuracy in localizing tumors, due to biopsy errors, and inaccuracy in imaging of $\mathrm{PCa}$; 2) difficulty of determining treatment success: following radical treatment the most sensitive measure of success is a near undetectable PSA value. However, focal treatment spares much of the normal glandular tissue, which also produces PSA, eliminating this measure of treatment efficacy; 3) lack of proven ablation techniques: the ablative procedure used must be reliable, safe, and its range of ablative effect precisely quantifiable.

In this pilot study, the feasibility of focally applying LDR-PB for treatment of PCa in selected patients was investigated. Our study protocol includes tumor localization and treatment planning based on a densely performed template-guided trans-perineal biopsy, and the use of multi-parametric MRI to assess for significant disease. This paper reports preliminary clinical results of this pilot project.

\section{Material and methods}

\section{Patient population and eligibility criteria}

The study population consists of 17 patients with favorable risk prostate adenocarcinoma, recruited between August 2013 and October 2016, who were suitable for or were on AS (mean age: 61.75 years; range: 47-73 years). The mean PSA level upon entry was $6.3 \mathrm{ng} / \mathrm{ml}$ (median: $5.1 \mathrm{ng} / \mathrm{ml}$; range: $0.35-15 \mathrm{ng} / \mathrm{ml}$ ), and mean prostate volume was $38.5 \mathrm{ml}$ (median: 33.5; range: 17.9-81.2 ml).

Initial diagnosis was made on standard trans-rectal ultrasound (TRUS) guided prostate biopsy (hereinafter referred to as TRUS biopsy) with no fewer than 6 cores taken. Eligibility at entry included $\leq 2$ positive cores from only one lobe, with clinical tumor stage $\leq \mathrm{T} 2 \mathrm{a}$ and Gleason score $(\mathrm{GS}) \leq 3+4$, PSA $\leq 10 \mathrm{ng} / \mathrm{ml}$, and no prior radiotherapy to the pelvis.

Institutional ethics approval was acquired and patient consent was obtained.

\section{Study outline}

Figure 1 illustrates the study schema. Upon consent, eligible participants underwent multi-parametric magnetic resonance imaging (mpMRI). Results were reviewed

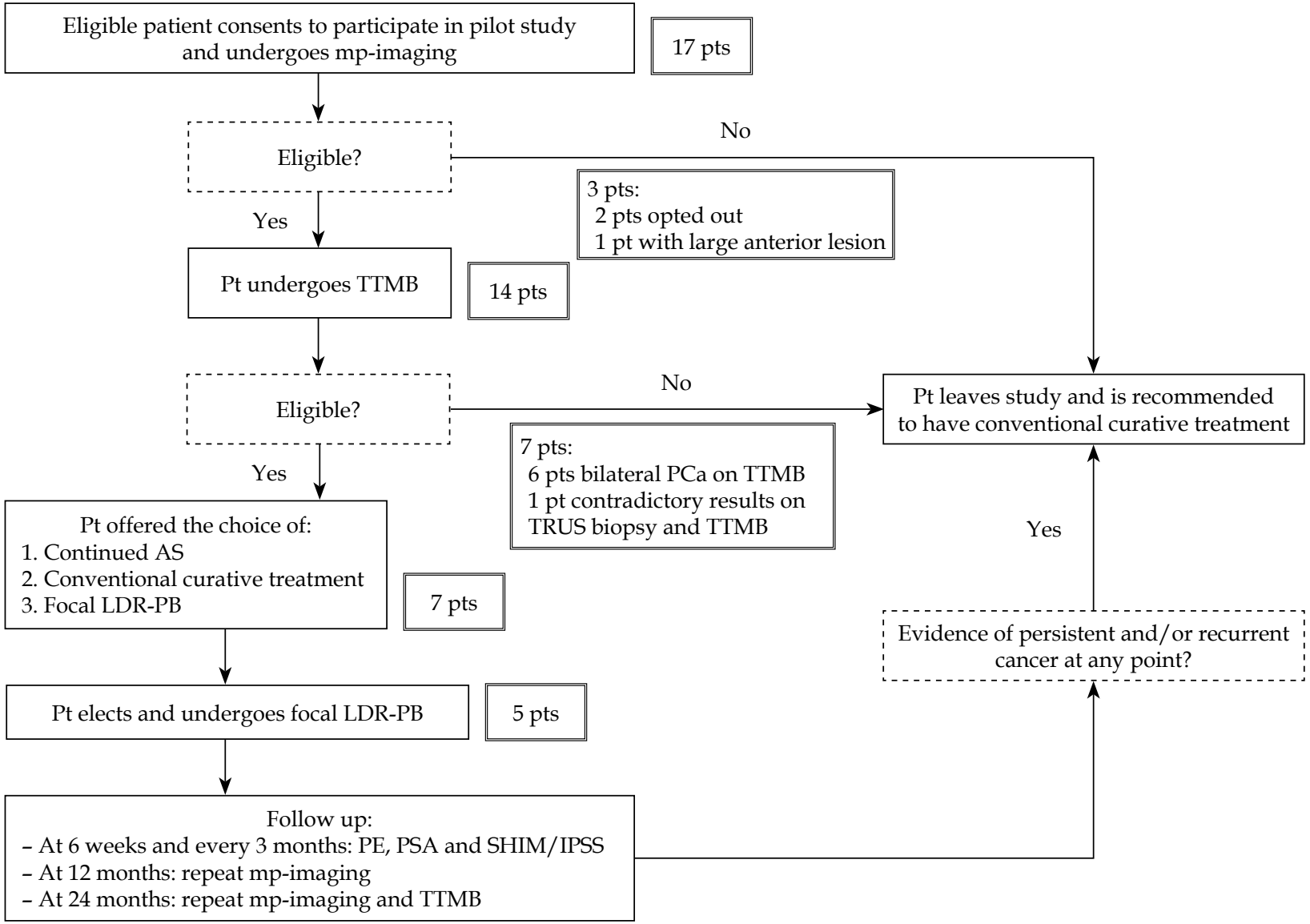

mp-imaging - multi-parametric imaging, TTMB - trans-perineal template-guided mapping biopsy, AS - active surveillance, LDR-PB - low-dose-rate prostate brachytherapy, PE - physical examination, PSA - prostate specific antigen, TRUS - trans-rectal ultrasound, SHIM - Sexual Health Inventory for Men, IPSS International Prostate Symptom score

Fig. 1. Study schema. The number of patients at each stage of the study is shown in double-bordered boxes 
by a single radiologist and suspicious regions were identified along with a PI-RADS score $[19,20]$. Those with no extra-capsular extension, neurovascular invasion, or positive lymph nodes on imaging, and a PI-RADS score $\leq 4$ out of 5 , underwent trans-perineal template-guided mapping biopsy (TTMB) to localize cancer. TTMB was performed in the surgical suite with the patient in a dorsal lithotomy position and under general or spinal anesthesia. The focal treatment was planned on the basis of the pathology findings from TTMB, and was carried out for eligible patients who opted to remain in the study.

Details of the imaging and TTMB procedures, treatment planning and execution, post-implant dosimetry, and follow-up are presented in the sections below.

\section{Multi-parametric magnetic resonance imaging}

Multi-parametric magnetic resonance imaging ( $\mathrm{mp}$ MRI) was performed using a Philips Achieva 3.0T MRI scanner (Philips Healthcare), located at the University of British Columbia MRI Research Centre [21], with a six element cardiac phased-array coil. Multi-parametric magnetic resonance imaging consisted of axial and coronal T2-weighted fast spin-echo images, diffusion-weighted imaging (DWI), and dynamic contrast-enhanced (DCE) imaging. DWI data were collected with $b$ factors $(0,600$, $1000)$ or $(0,100,500,1000) \mathrm{s} / \mathrm{mm}^{2}$, from which apparent diffusion coefficient (ADC) maps were computed. DCE studies were performed using a 3D T1-weighted spoiled gradient echo sequence. First, proton density (PD) imaging was performed to allow calculation of the contrast agent concentrations in the prostate. Next, T1-weighted images were acquired prior to, and following a bolus intravenous injection of Gd-DTPA (Magnevist, Berlex Canada).

\section{Trans-perineal template-guided mapping biopsy}

Trans-perineal template mapping biopsy (TTMB) was performed using the standard LDR-PB setup with a $5 \mathrm{~mm} \times 5 \mathrm{~mm}$ brachytherapy template (Figure 2). First, a TRUS 'volume study' was performed, consisting of $5 \mathrm{~mm}$ spaced axial TRUS images of the prostate overlaid with a grid calibrated to match the brachytherapy template. Core mapping and treatment planning is done on these images. Next, depending on the volume of the prostate, 20-50 cores were extracted from selected grid locations using a Bard biopsy gun (Bard Inc., NJ, USA) with $18 \mathrm{G} \times 25 \mathrm{~cm}$ needles and a $22 \mathrm{~mm}$ throw. Our goal was to uniformly sample the prostate while limiting the number of extracted cores to avoid excessive trauma to the gland, and to avoid sensitive regions such as the urethra. Where the length of the prostate was greater than the throw of the gun, two cores were extracted, first proximal and then distal. Core grid locations and their craniocaudal depths (with a precision of $5 \mathrm{~mm}$ - the volume study image spacing) were recorded.

The samples were prepared immediately by two histology technicians, with the distal ends marked, and then sent for conventional embedding and H\&E staining. The biopsy samples were reviewed by a single pathologist. For every core, the presence of cancer, Gleason score, tumor location and length of involvement within

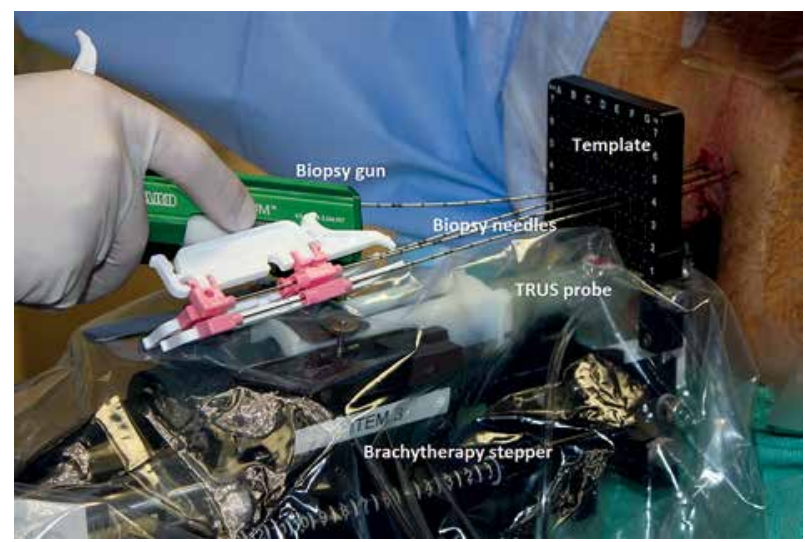

Fig. 2. Trans-perineal template-guided mapping biopsy setup

the core, along with other anomalies such as high-grade prostatic intraepithelial neoplasia (PIN) and prostatitis were reported.

Patients were given intraoperative prophylactic antibiotics (1 gram cefazolin IV) and were discharged from day care when they were able to void and had recovered from anesthesia, approximately 1-2 hours after the procedure. Trans-perineal template mapping biopsy required 60-90 minutes operating room time, including patient preparation.

Patients with TTMB indicating unilateral PCa were deemed eligible for and were offered focal LDR-PB, in addition to standard options of remaining on AS or radical treatment.

\section{Treatment planning}

The general approach to planning focal LDR-PB followed our standard practice for whole gland brachytherapy, with some adaptations to limit the dose deposition to smaller targets, thereby providing additional sparing of the urethra, bladder neck, rectum, and the nerves responsible for erectile function, as well as the majority of healthy glandular prostate epithelium. The focal gross tumor volume (F-GTV) was defined as TTMB derived cancer locations. These were mapped onto the TRUS volume study using the localization information recorded during TTMB. The focal clinical target volume (F-CTV) was defined as the F-GTV, plus a $5 \mathrm{~mm}$ margin in all three dimensions, cropped to the prostate boundary. The focal planning target volume (F-PTV) was drawn based on the F-CTV, and included the surrounding un-sampled grid locations up to grid points with negative biopsy results. Such margins were added to account for potentially undetected tumors in un-sampled regions, and F-GTV localization errors.

Extra-prostatic extension of the F-PTV was allowed up to $4-5 \mathrm{~mm}$ from the prostate boundary except posteriorly. The rectum was delineated from $1 \mathrm{~cm}$ superior to the base to $1 \mathrm{~cm}$ inferior to the apex. As urethral contrast was not used during imaging, a $5 \mathrm{~mm}$ radius urethral avoidance structure was contoured according to [22].

Contouring was performed with the MIM Symphony (MIM Software, Cleveland, OH, USA) and planning was 


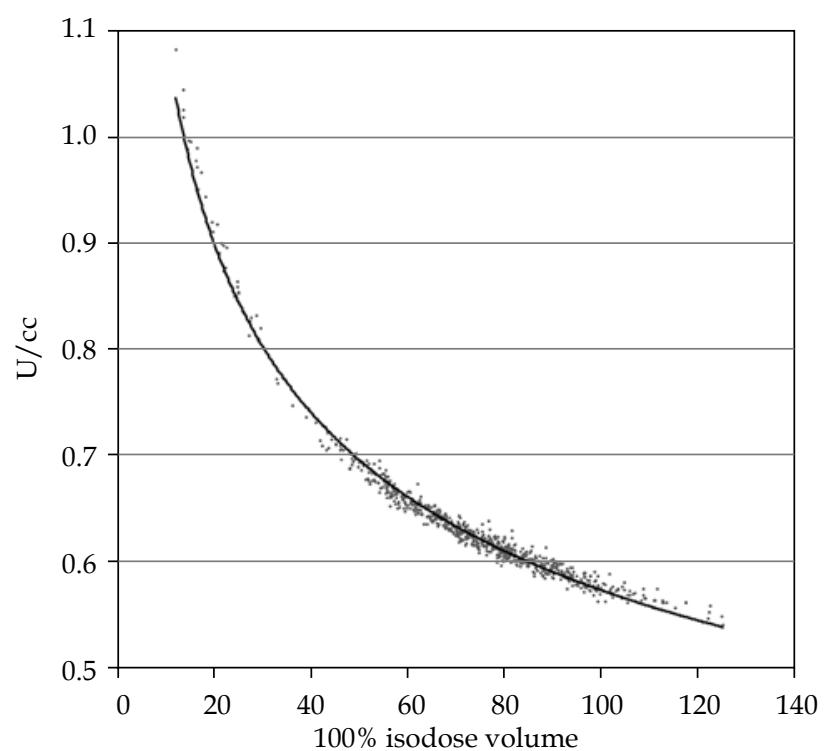

Fig. 3. Total air kerma strength density as a function of $100 \%$ isodose volume for plans created in our center using $0.4 \mathrm{U}$ sources

performed with the VariSeed 8.0 planning system (Varian Medical Systems, CA, USA).

Because focal therapy PTV's are generally smaller than typical whole gland treatment volumes, a higher total source strength density $\left(\mathrm{U} / \mathrm{cm}^{3}\right)$ is required to deliver the prescription dose [23]. Figure 3 illustrates the total air kerma strength density as a function of the $100 \%$ isodose volume for plans created in our center using $0.4 \mathrm{U}$ sources. Therefore, in order to limit the F-PTV seed density, we employed a dual air kerma strength planning approach intended to achieve adequate dose coverage with reasonable (e.g. $\sim 0.7 \mathrm{~cm}$ ) inter-source spacing, but also avoiding high strength sources near the rectum and urethra. The resulting plans delivered a prescription dose of 144 Gy to the F-PTV using a combination of low strength sources $(0.4 \mathrm{U})$, placed towards the prostate center, and high strength sources (0.8-0.9 U), placed at the prostate periphery (except posteriorly near the rectum), with the goal of meeting dosimetric constraints: F-PTV $V_{100} \geq 98 \%$, and 55\% < F-PTV V $V_{150}<65 \%$ of structure volumes. The exception was patient \#17, in which due to the very small F-PTV size, a single high air kerma strength plan $(0.732 \mathrm{U})$ was employed. Dose to the rectum and urethra were minimized as much as possible (urethra $\mathrm{V}_{100} \leq 30 \%$, and rectum $\mathrm{V}_{100} \leq 5 \%$ of structure volumes). The dose to the organs at risk varied somewhat from patient to patient depending on their proximity to the F-CTV, but were lower than the doses that would be received in whole gland LDR-PB. A TG43 point source model with anisotropy factors was used for the dose calculations. Stranded source trains with Oncoseed 6711 sources (Oncura Inc., Arlington Heights, IL, USA) were used, and implantation was performed under TRUS and fluoroscopy guidance as in whole gland LDR-PB. Details of the planning procedure are presented in [24].

Post-implant day-0 CT was performed 2-3 hours post implant. Structures (prostate, urethra, bladder, and rectum) were manually outlined, and source identification (i.e. the identification of low and high source strengths) was performed using an in-house automatic plan reconstruction algorithm [25]. In this unique algorithm, sources identified on day- 0 CT are registered to the treatment plan. By finding the correspondence between the sources on day-0 CT and the treatment plan, and considering that the source activities are known in the treatment plan, it is possible to identify the activity of each source on day-0 CT.

In standard LDR-PB, the CTV (i.e. the prostate boundary) is fairly visible and is outlined on day-0 CT images for post-implant dosimetry. Since in focal therapy, the F-CTV is an irregular shaped region with no visible boundaries, it is not possible to directly identify it on day-0 CT images. We devised a two-step method to map TRUS planning contours onto day-0 CT images. First, the TRUS planning contours were mapped onto pre-treatment MRI images using an in-house 3D model-based deformable registration algorithm with a constant elasticity map [26]. This step is performed to correct for the posterior warping of the prostate in TRUS images due to the presence of the probe, which is absent in MRI and day-0 CT. Next, the resulting contours were mapped onto day-0 CT using manual rigid registration between MRI and day-0 CT. Dose metrics, as listed in Table 2 under post-implant dosimetry, were then computed for the F-CTV, urethra and rectum using the VariSeed software.

\section{Follow-up}

Follow-up of focally treated patients includes physical examination, PSA, TTT measurements, IPSS (International Prostate Symptom score), and SHIM (Sexual Health Inventory for Men) questionnaires at 6 weeks and every 3 months post-treatment, up to 24 months, and every 6 months thereafter; mpMRI repeated 1 and 2 years post-treatment, and TTMB at 2 year follow-up. If evidence of persistent disease is observed, patients are recommended to proceed with surgical extirpation.

\section{Results}

Table 1 describes the TTMB results, eligibility, and the treatment of choice for the study population. The number of patients at each stage of the study is shown in Figure 1. A total of 17 patients was enrolled in the study, three of whom did not continue after mpMRI (patients \#3, \#7, and \#14).

Out of the 14 remaining patients, seven were considered ineligible after TTMB: TTMB results indicated bilateral disease in six patients. One (patient \#2) was advised to exit the study due to contradictory results. In this patient, TTMB and 3 previous TRUS biopsies reported tumor in opposite lobes while nothing suspicious was observed on mpMRI. Seven were eligible for focal LDR-PB, five of whom received focal LDR-PB.

No complications were observed after TTMB. Twelve repeat mpMRI has been performed on three focally treated patients (patients \#5, \#6, and \#13). At 12 months, no suspicious lesion is seen on the repeat mpMRI of patients \#5 and \#13; patient \#6 shows a small $\left(\leq 0.25 \mathrm{~cm}^{3}\right)$ PI-RADS 3 area in the treated lobe $(>0.3 \mathrm{cc}$, peripheral zone, posterior base). From the 24 month repeat mpMRI 
Table 1. Multi-parametric magnetic resonance imaging and trans-perineal template-guided mapping biopsy results, and the treatment of choice for the study population

\begin{tabular}{|c|c|c|c|c|c|c|c|c|c|c|}
\hline \multirow[t]{2}{*}{ Patient } & \multicolumn{2}{|c|}{ mpMRI } & \multicolumn{4}{|c|}{ TTMB } & \multirow[b]{2}{*}{$\begin{array}{l}\text { Highest } \\
\text { GS from } \\
\text { pre-en- } \\
\text { rollment } \\
\text { TRUS } \\
\text { biopsy }\end{array}$} & \multirow{2}{*}{$\begin{array}{l}\text { Eligible } \\
\text { for focal } \\
\text { LDR-PB? }\end{array}$} & \multirow[t]{2}{*}{ Treatment* } & \multirow{2}{*}{$\begin{array}{l}\text { Reason for } \\
\text { ineligibility }\end{array}$} \\
\hline & $\begin{array}{l}\text { Prostate } \\
\text { vol. (cc) }\end{array}$ & $\begin{array}{l}\text { Highest } \\
\text { PI-RADS }\end{array}$ & $\begin{array}{l}\text { No. cores } \\
\text { extracted }\end{array}$ & $\begin{array}{c}\text { No. } \\
\text { positive } \\
\text { cores }\end{array}$ & $\begin{array}{l}\text { Highest } \\
\text { GS }\end{array}$ & $\begin{array}{c}\text { Total PCa } \\
\text { extent } \\
\text { within } \\
\text { cores } \\
(\mathrm{mm})\end{array}$ & & & & \\
\hline 1 & 20.0 & 3 & 28 & 2 & $3+4$ & 6.5 & $3+4$ & $\mathrm{~N}$ & LDR-PB & Bilateral PCa \\
\hline 2 & 17.9 & $\begin{array}{l}\text { No lesion } \\
\text { found }\end{array}$ & 32 & 2 & $3+3$ & 4 & $3+3$ & $\mathrm{~N}$ & AS & $\begin{array}{l}\text { Contradictory } \\
\text { biopsy results }\end{array}$ \\
\hline 3 & 81.2 & 4 & - & - & - & - & $3+3$ & Withdrew & AS & \\
\hline 4 & 26.5 & 2 & 24 & 2 & $3+3$ & 5.2 & $3+4$ & Y & $\mathrm{RP}$ & \\
\hline 5 & 63.5 & 3 & 48 & 2 & $3+3$ & 4 & $3+3$ & Y & Focal LDR-PB & \\
\hline 6 & 29.7 & 3 & 29 & 3 & $3+3$ & 7.1 & $3+3$ & $\mathrm{Y}$ & Focal LDR-PB & \\
\hline 7 & 50.7 & 3 & - & - & - & - & $3+3$ & Withdrew & $\begin{array}{c}\text { Status } \\
\text { unknown }\end{array}$ & \\
\hline 8 & 48.5 & 3 & 38 & 5 & $4+3$ & 15 & $3+4$ & N & LDR-PB & Bilateral PCa \\
\hline 9 & 29.2 & 2 & 32 & 8 & $3+3$ & 41.5 & $3+3$ & N & AS & Bilateral PCa \\
\hline 10 & 20.2 & 3 & 22 & 5 & $3+3$ & 16 & $3+3$ & N & LDR-PB & Bilateral PCa \\
\hline 11 & 66.0 & 3 & 38 & 3 & $3+4$ & 4 & $3+4$ & $\mathrm{~N}$ & LDR-PB & Bilateral PCa \\
\hline 12 & 34.3 & 3 & 23 & 5 & $3+3$ & 7 & $3+3$ & $\mathrm{~N}$ & LDR-PB & Bilateral PCa \\
\hline 13 & 43.0 & 4 & 30 & 2 & $3+3$ & 4 & $3+3$ & Y & Focal LDR-PB & \\
\hline 14 & 19.1 & 5 & - & - & - & - & $3+4$ & $\mathrm{~N}$ & AS & $\begin{array}{l}\text { Large PI-RADS } \\
5 / 5 \text { anterior } \\
\text { lesion on } \\
\text { mpMRI. TTMB } \\
\text { not performed }\end{array}$ \\
\hline 15 & 22.5 & 4 & 22 & 3 & $3+4$ & 6.5 & $3+4$ & Y & Focal LDR-PB & \\
\hline 16 & 48.9 & 3 & 41 & 5 & $3+4$ & 8.5 & $3+4$ & Y & AS & \\
\hline 17 & 33.5 & 4 & 28 & 3 & $3+4$ & 3 & $3+4$ & Y & Focal LDR-PB & \\
\hline
\end{tabular}

${ }^{*}$ As of December 2016

mPMRI - multi-parametric magnetic resonance imaging, TTMB - trans-perineal template mapping biopsy, AS - active surveillance, LDR-PB - low-dose-rate prostate brachytherapy, RP-radical prostatectomy, $P C a$ - prostate cancer, GS - Gleason score

performed on patients \#5 and 6, a PI-RADS 3 area is reported in the treated region of patient \#5 $(>0.9 \mathrm{cc}$, central zone, lateral base, and mid-gland), whereas no suspicious region is seen in patient \#6.

At 24 months, two patients (patient \#5 and \#6) received repeat TTMB. In comparison to the tumor present in the original pre-treatment TTMB (Figure 4A), pathology for the repeat TTMB showed no evidence of residual viable tumor. However, many of the biopsy cores for both patients showed glandular atrophy and degenerative-type nuclear atypia, consistent with the effects of LDR-PB. These findings were apparent on both the treated and untreated sides, but to a much greater extent on the treated side (Figure 4B, C). The benign nature of these atrophic glands is supported by the presence of an intact myoepithelial layer staining positive for the myoepithelial immunohistochemistry marker p63 (Figure 4D). In a few of these biopsy cores, localizing to similar areas showing positivity for tumor in the original TTMB biopsies, there were tiny foci of haphazardly arranged atrophic glands (Figure 4E), and a few glands showed possible absence of myoepithelial cells based on immunohistochemical staining for p63 (Figure 4F). Therefore, it was difficult to unequivocally determine if these represented atrophic tumor glands or normal glands. However, direct comparison of the morphology of these atypical glands with tumor glands in the original biopsies showed no histological resemblance. Therefore, repeat TTMB biopsies in both patients were diagnosed as benign, and classified as showing complete histologic response to radiotherapy.

\section{Multi-parametric magnetic resonance imaging and template-guided mapping biopsy results}

In total, 435 TTMB cores were extracted, $1 \pm 0.3$ cores per cc of prostate volume (range: $0.6-1.8$ ), out of which 50 
Table 2. Planning information and post-implant dosimetry for the focal low-dose-rate brachytherapy patients

\begin{tabular}{|c|c|c|c|c|c|c|}
\hline & & Patient \#5 & Patient \#6 & Patient \#13 & Patient \#15 & Patient \#17 \\
\hline \multicolumn{7}{|c|}{ Planning dosimetry } \\
\hline \multicolumn{2}{|l|}{ F-PTV vol. [cc] } & 12.9 & 12.8 & 9.3 & 9.3 & 5.5 \\
\hline \multicolumn{2}{|c|}{ F-PTV vol./Prostate vol. } & 0.20 & 0.43 & 0.22 & 0.41 & 0.16 \\
\hline \multicolumn{2}{|c|}{ Source strength [U] } & $0.868,0.417$ & $0.941,0.417$ & $0.941,0.417$ & $0.732,0.413$ & 0.732 \\
\hline \multicolumn{2}{|c|}{$\begin{array}{l}\text { Total source strength/ } \\
100 \% \text { isodose volume }\end{array}$} & 0.92 & 0.94 & 1.12 & 1.09 & 1.31 \\
\hline \multicolumn{2}{|l|}{ \# needles (H, L) } & 5,4 & 4,4 & 4,2 & 4,4 & 6 \\
\hline \multicolumn{2}{|l|}{ \# sources $(\mathrm{H}, \mathrm{L})$} & 15,14 & 14,13 & 13,7 & 13,12 & 15 \\
\hline \multicolumn{2}{|c|}{ Mean seeds/needle } & 3.23 & 3.38 & 3.34 & 3.13 & 2.5 \\
\hline \multirow[t]{3}{*}{ F-PTV } & $\mathrm{V}_{100}[\%]$ & 88.6 & 94.9 & 96.5 & 95.4 & 98.0 \\
\hline & $\mathrm{D}_{90}[\mathrm{~Gy}]$ & 137.5 & 158.6 & 165.3 & 162.9 & 172.6 \\
\hline & $\mathrm{V}_{150}[\%]$ & 49.2 & 64.1 & 66.9 & 63.7 & 68.2 \\
\hline \multirow[t]{2}{*}{ Rectum } & $\mathrm{D}_{1 \mathrm{cc}}[\mathrm{Gy}]$ & 44.7 & 86.0 & 33.5 & 60.5 & 21.8 \\
\hline & $\mathrm{V}_{100}[\mathrm{cc}]$ & 0.0 & 0.0 & 0.0 & 0.0 & 0.0 \\
\hline \multirow[t]{2}{*}{ Urethral avoid } & $\mathrm{V}_{125}[\mathrm{cc}]$ & 0.3 & 0.0 & 0.0 & 0.3 & 0.0 \\
\hline & $\mathrm{V}_{30}[\%]$ & 88.9 & 94.4 & 83.7 & 100 & 74.9 \\
\hline \multicolumn{7}{|c|}{ Post-implant dosimetry } \\
\hline \multirow[t]{2}{*}{ F-CTV } & $\mathrm{V}_{100}[\%]$ & 88.8 & 94.0 & 92.8 & 92.3 & 84.3 \\
\hline & $\mathrm{V}_{150}[\%]$ & 57.1 & 63.3 & 62.8 & 61.5 & 50.2 \\
\hline \multirow[t]{2}{*}{ Rectum } & $\mathrm{D}_{1 \mathrm{cc}}[\mathrm{Gy}]$ & 50.2 & 69.5 & 34.9 & 81.6 & 39.3 \\
\hline & $\mathrm{V}_{100}[\mathrm{cc}]$ & 0.0 & 0.0 & 0.0 & 0.1 & 0.0 \\
\hline \multirow[t]{2}{*}{ Urethra } & $\mathrm{V}_{125}[\mathrm{cc}]$ & 0.0 & 0.0 & 0.0 & 0.0 & 0.0 \\
\hline & $\mathrm{V}_{30}[\%]$ & 61.3 & 88.3 & 15.4 & 88.1 & 64.4 \\
\hline
\end{tabular}

F-PTV-focal planning target volume, $(H, L)$ - high strength, low strength, F-CTV-focal clinical target volume, $V_{100 \%}, V_{150 \%}, V_{125 \%}, V_{30 \%}-$ volume of the anatomic volume receiving $100 \%, 150 \%, 125 \%, 30 \%$ of the prescribed dose, $D_{90}$ - minimum prescribed dose received by $90 \%$ of the anatomic volume, $D_{1 c c}-$ minimum dose to the most exposed $1 \mathrm{~cm}^{3}$

(11\%) were positive for PCa. Out of the 14 patients who received both TTMB and mpMRI, 11 had PI-RADS $\geq 3$ lesions on mpMRI.

An indication of unilateral disease based on TRUS biopsy is required upon entry, however, six patients were found to have bilateral PCa on TTMB. Three bilateral PCa cases were detected on mpMRI, two of which were indeed bilateral on TTMB.

One patient (patient \#4) received RP. Final pathology from the RP indicates GS $3+4$ mostly on the left posterolateral and anterolateral inferior half, and left posterior portion of the apex. This concurs with TRUS biopsy and TTMB results. However, TTMB indicated a maximum GS of $3+3$, whereas pathology results from RP detected $3+4$.

We performed a sector based comparison between mpMRI-based radiologist findings and the results of TTMB. Twelve sectors were considered: left, right, anterior and posterior in the base, mid-gland and apex [27]. Multi-parametric magnetic resonance imaging findings and TTMB results were manually mapped onto the corresponding 12 sectors. If we consider only PI-RADS $\geq 3$ lesions, the sensitivity (true positives) of mpMRI in local- izing PCa in our cohort was $37.5 \%$. The specificity, positive predictive value, negative predictive value, and accuracy of mpMRI-based PCa detection were $94.4 \%, 63.2 \%$, $86.6 \%$, and $83.9 \%$, respectively. However, among the GS 7 lesions ( $n=12$ cores), 10 cores were correctly identified on mpMRI, suggesting a higher sensitivity to GS $\geq 7$, as expected.

\section{Treatment planning}

Planning information and post-implant dosimetry for the five focally treated patients are provided in Table 2 . Figure 5 shows treatment plans at three levels: the base, mid-gland, and apex for three cases. F-PTV coverage in patient \#5 was compromised due to significant overlap with the urethral avoid, and the desire to spare this region.

\section{Prostate specific antigen, International Prostate Symptom score, and Sexual Health Inventory for men results}

Prostate specific antigen kinetics of patients treated with focal LDR-PB, whole gland LDR-PB and those on 
A

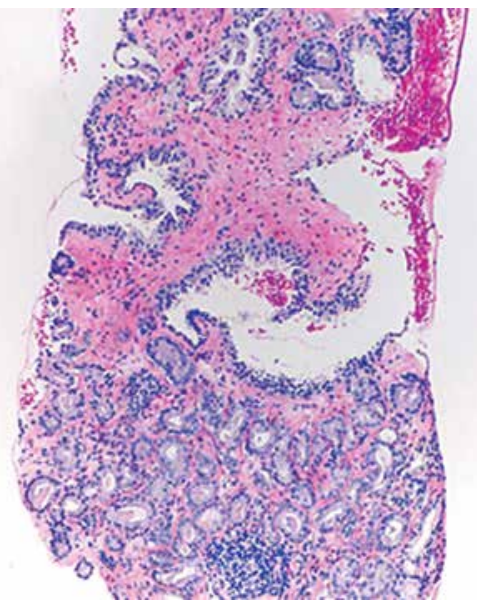

C

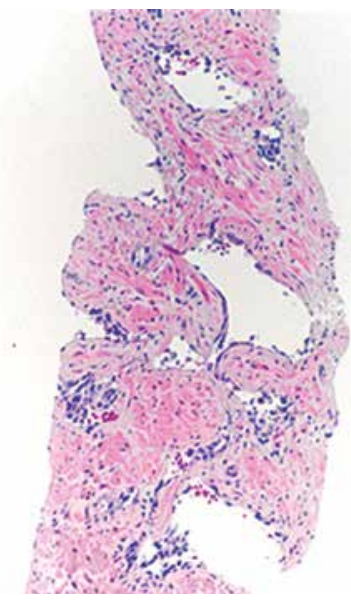

E

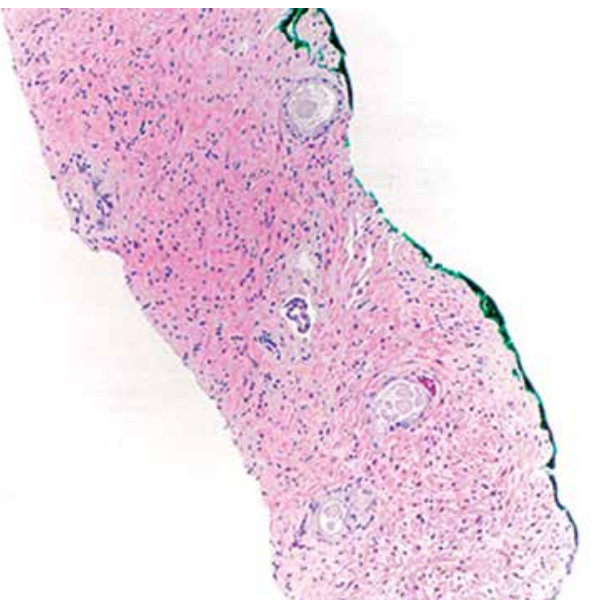

B

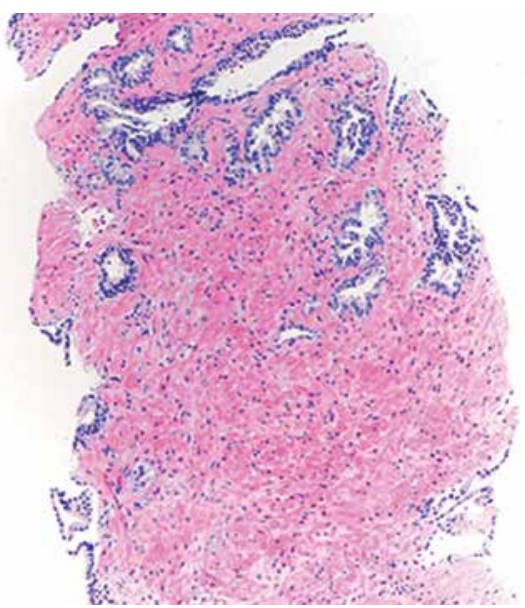

D

$\mathbf{F}$
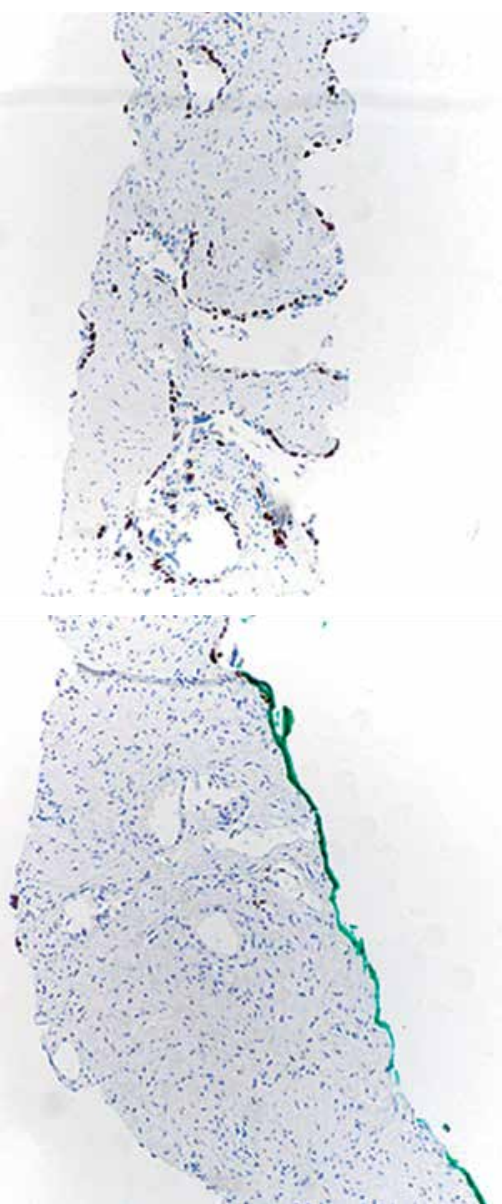

Fig. 4. A) Original pre-treatment trans-perineal template mapping biopsy (TTMB) biopsy from patient \#5 demonstrating Gleason score $3+3=6$ adenocarcinoma demonstrated in the lower half of the image, with benign gland present in the top half of the image. B) Post-treatment TTMB biopsy from untreated half of prostate, showing moderate glandular atrophy. C) Post-treatment TTMB biopsy from treated half of prostate, showing marked glandular atrophy. D) p63 immunohistochemistry demonstrating intact myoepithelial layer around atrophic glands. E) Tiny foci of haphazardly arranged glands in neighborhood of the site of identified tumor in original biopsy. F) p63 immunohistochemistry showing only rare myoepithelial cells associated with this atypical focus

active surveillance (one of whom underwent RP) are plotted in Figure 6A-C (median follow-up 24, 30, 27 months, respectively), with baseline PSA defined as values immediately prior to TTMB.

Post-operative PSA in LDR-PB patients are declining. There is no specific trend seen in PSA values in those treated with focal LDR-PB and AS. Less decrease in post-operative PSA values of patients treated with focal LDR-PB compared to those of whole-gland LDR-PB is expected due to the presence of untreated glandular tissue.

Figures 6D-G demonstrate the SHIM and IPSS trends at baseline (immediately prior to focal LDR-PB) and post 

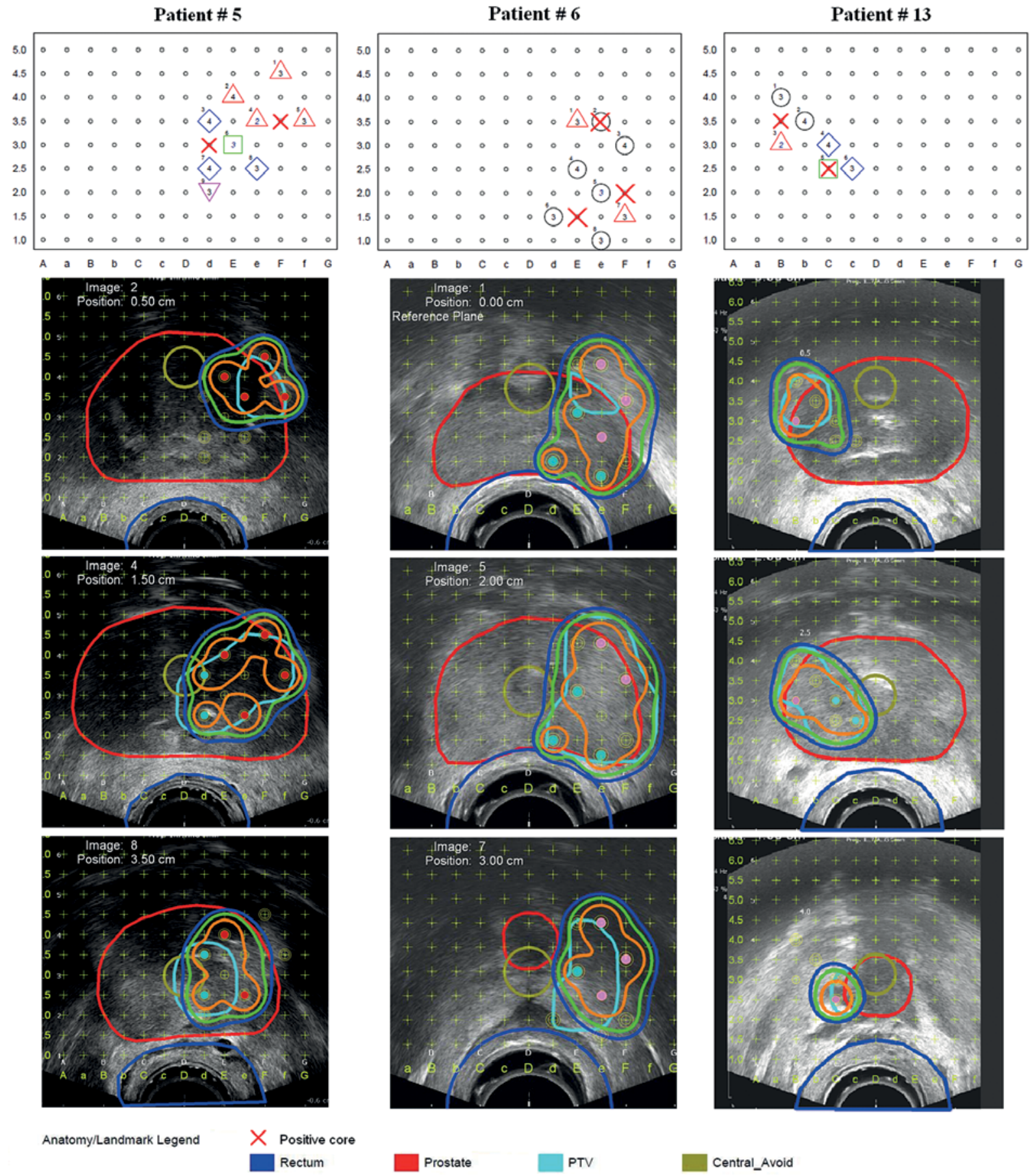

Prostate

PTV

Central_Avoid

Isodose Legend

Gy [\% of Prescription Dose]

$216.00[150.00 \%]$

$144.00[100.00 \%]$

$115.20[80.00 \%]$

Fig. 5. Implanted dual strength plans for three focal LDR-PB patients showing central low strength (cyan) and peripheral high strength (pink/red) sources. In the needle distribution (row 1), the icons circle, triangle, square, diamond, and inverted triangle represent needles planned to retraction planes $0 \mathrm{~cm}, 0.5 \mathrm{~cm}, 1 \mathrm{~cm}, 1.5 \mathrm{~cm}$, and $2 \mathrm{~cm}$ inferior to the base. The coordinates of the positive cores are marked by a cross. Source distributions are shown for the base, mid-gland, and apex (rows 2-4)

treatment (median SHIM follow-up 21 and 26 months, and IPSS follow-up 24 and 26 months for the focal LDR-PB and whole-gland LDR-PB groups, respectively). Higher SHIM and lower IPSS scores indicate better sexual and urinary health, respectively.
International Prostate Symptom score and SHIM scores in focal LDR-PB patients are relatively stable. Two patients (patient \#5 and \#13) had severe erectile dysfunction both prior to and post focal treatment. The other patient (patient \#6) showed very good SHIM and IPSS 


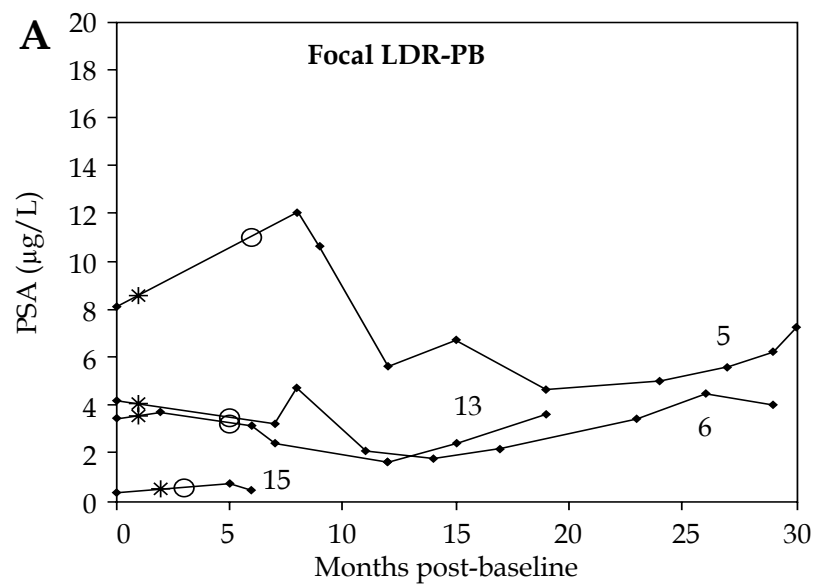

Fig. 6. PSA kinetics of patients treated with (A) focal LDR-PB, (B) whole gland LDR-PB, and (C) those on active surveillance (AS) or radical prostatectomy (RP). Baseline is the time of PSA measurement before, but closest to TTMB. SHIM scores for (D) focal LDR-PB (with more than 6 months of follow-up) and (E) whole gland LDR-PB patients. IPSS scores for (F) focal LDR-PB and (G) whole gland LDR-PB patients. Baseline values are scores collected prior to treatment. Time of TTMB is marked by an asterisk $\left(^{*}\right)$ and time of treatment is marked by a circle $(\circ)$. Data points are marked as dots or stars. Plots are labeled with the corresponding patient number
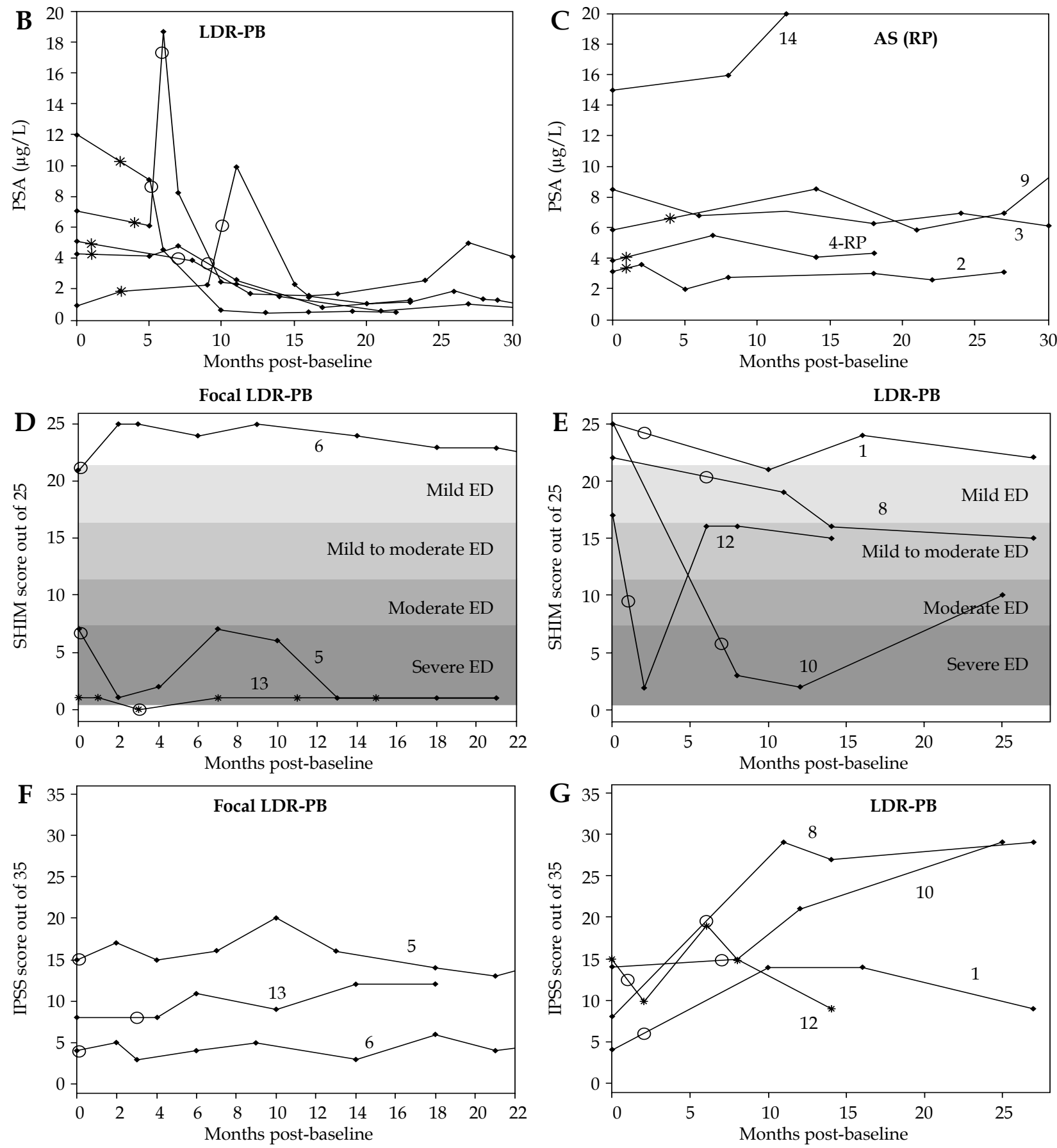
scores both prior to and after focal LDR-PB (patient \#15 and 17 are recently treated, and hence, are not included in the plots). In whole gland LDR-PB patients, SHIM scores have either remained unchanged or worsened, and IPSS scores have increased compared to baseline and have not returned to baseline for two of the four patients.

No gastrointestinal, genitourinary, or grade $3+$ toxicity (LENT/SOMA scale [28]) was observed in the focal LDR-PB patients immediately after treatment or at follow-up.

\section{Discussion}

Few groups have investigated the use of LDR-PB for focal treatment $[29,30]$, and long-term outcomes of focal LDRPB are unknown. To date, based on clinicaltrials.gov, only five groups are actively recruiting patients for focal prostate brachytherapy [31,32,33,34,35], one being our center.

A main limitation of this study is the small population size. While these preliminary results can provide valuable insights toward a larger study, we understand that it is not possible to draw meaningful conclusions.

Another limitation of this study and focal LDR-PB in general, is the lack of enough data on what the appropriate approach should be in case of recurrence or spread of disease. We do not have direct experience, as patients who received repeat TTMB at 24 months did not show any sign of recurring PCa. In whole gland treatment, focal salvage LDR-PB has been suggested for recurring PCa after LDR-PB or prostatectomy [36,37]. If disease is confined to the contralateral side, we might suggest a second focal LDR-PB on that side.

Post-implant dosimetry requires accurate localization of the implanted sources and delineation of the targeted area (i.e. F-CTV). We devised a two-step approach to map the TRUS-based planning F-CTV onto day-0 CT, with the help of pre-treatment MRI images. A combination of contour-based automatic deformable registration and imageand contour-based manual rigid registration was used. The accuracy of this approach relies on accurate delineation of the boundaries. Furthermore, manual registration is observer dependent. A possibly more reliable approach would be the use of fiducial markers implanted in the prostate a few weeks before imaging (to allow adequate time for the markers to fixate within the tissue). This will, however, require an additional procedure in the operating room.

One of the contributions of this study is the use of dual source strength planning with the purpose of addressing issues related to treating smaller (e.g. focal) targets. In a small target, single strength low activity seeds result in overly dense plans, which are difficult to implant; with high activity seeds, it is difficult to deliver the prescribed dose while avoiding the risk of overdosing critical regions, especially when considering the possibility of seed misplacement. With a dual source strength planning strategy, we were able to achieve the required dose distribution with a reasonable number of needles. In a separate article, we studied the characteristics and robustness to source displacement in dual source strength plans vs. single high, intermediate, and low source strength plans for focal LDR-PB. We showed that the use of dual source strength treatment plans for focal LDR-PB is possibly the practical solution for limiting the density of sources required to deliver the prescribed dose, while limiting proximity of high strength sources to organs at risk [24].

Trans-rectal ultrasound biopsies are known to be unreliable in localization and risk stratification $[38,39,40]$. In this study, 6 out of the 17 patients with unilateral PCa on TRUS biopsy were deemed ineligible on TTMB for focal LDR-PB due to bilateral disease. Template-guided mapping biopsy has been proposed as a more accurate approach to re-staging prior to focal therapy with few and minor complications $[41,42,43]$. It has the advantage of providing an accurate three dimensional map of PCa, which is reproducible for focal treatment and follow-up biopsies, and is particularly attractive for focal LDR-PB, since it uses the same system applied for LDR-PB volume study and quality assurance. Based on an analysis similar to that in [44], at a $1 \mathrm{~cm}$ grid spacing (similar to our TTMB core density of $1 \pm 0.3$ cores/cc), a 0.5 cc tumor - the threshold for tumor significance [45] - has a $>85 \%$ probability of being detected. Disadvantages of TTMB include higher cost and complexity. It requires operating room time with regional or general anesthesia, and processing of a large number of biopsy cores. However, it can be particularly useful in detecting anterior tumors, which are present in up to $30 \%$ of cases [46], but are often missed by TRUS biopsy. In our study, half of the positive cores were located in anterior half of the gland.

Multi-parametric magnetic resonance imaging-based sensitivity in localizing tumors was low in this study. We attribute this, compared to that in the literature [47, and references therein], to the lower-risk population used in this study, i.e. those on AS with a small tumor extent per core (mean \pm standard deviation $2.6 \pm 2.6 \mathrm{~mm}$; median: $2 \mathrm{~mm}$; range: $0.5-11 \mathrm{~mm}$ ), a majority of lesions identified as PI-RADS 3 ( 9 out of 17 cases), and GS of $3+3$ in 38 of the 50 cores $(76 \%)$. The location and aggressiveness of the tumors and the use of endorectal coil is shown to greatly affect the detection rates $[47,48,49]$. Furthermore, higher b-value DWI $\left(1400-2000 \mathrm{~s} / \mathrm{mm}^{2}\right)$ is recommended in PI-RADS v2 [19]. Finally, it is important to consider the inter- and intra-observer variability in manual interpretation of mpMRI. In this study, interpretation was performed by a single radiologist.

Studies on MRI and AS mainly concentrate on using MRI for detecting or ruling out clinically significant disease, which might prompt a change from AS to radical treatment. A systematic review paper on MR imaging in AS [50] summarizes that a lesion suspicious for prostate cancer is seen on MRI in roughly two-thirds of men suitable for AS. In our study, 9 of 14 patients had PI-RADS $\geq 3$ lesions confirmed by TTMB in a region negative for PCa by TRUS biopsy. In summary, our results suggest that mpMRI can be a useful screening tool for detecting significant GS $\geq 7$ PCa within the prostate, and for detecting bilateral disease for focal LDR-PB treatment, possibly eliminating the need for TTMB if bilateral PCa is found.

As a further step, we plan to combine and correlate imaging data collected prior to and after treatment with TTMB results. The current protocol includes the collection of MR elastography and mpTRUS data. Magnetic resonance elas- 
tography is performed as a part of the mpMRI procedure $[51,52]$ and mpTRUS, which also includes TRUS elastography that is collected immediately prior to TTMB $[53,54,55]$. A sufficiently accurate image-based cancer detection method (e.g. resulting in cancer maps from multi-modality, multi-parametric imaging) would have the potential to replace TTMB and TRUS biopsy for initial patient selection, and for monitoring the efficacy of treatment.

\section{Conclusions}

Our preliminary results suggest the feasibility of LDR-PB for focal treatment. In particular, it can be easily customized to treat a partial prostate volume with almost no modification to the standard treatment procedure, and appears to have a more favorable side effect profile. However, being at an early stage, great care should be taken in patient selection and implementation, and further studies on larger populations are required for better evaluation of the methods used and to assess long-term outcomes.

\section{Acknowledgement}

We would like to thank the BC Cancer Foundation for funding this study. Special thanks to the staff of BCCA Vancouver Centre and UBC 3T MRI Research Centre, and Dr. David Huntsman and Peggy Tsang at the Centre for Translational and Applied Genomics (CTAG) for assisting us throughout this study.

\section{Disclosure}

Dr. Mahdavi reports salary payment and Dr. Ng reports personal fees (for consultation on pathologic diagnoses) from the BC Cancer Foundation, during the conduct of the study. All other authors report no proprietary or commercial interest in any product mentioned or concept discussed in this article.

\section{References}

1. Crook JM, Gomez-Iturriaga A, Wallace K et al. Comparison of health-related quality of life 5 years after SPIRIT: Surgical Prostatectomy Versus Interstitial Radiation Intervention Trial. J Clin Oncol 2011; 29: 362-368.

2. Mohammed N, Kestin L, Ghilezan M et al. Comparison of acute and late toxicities for three modern high-dose radiation treatment techniques for localized prostate cancer. Int J Radiat Oncol Biol Phys 2012; 82: 204-212.

3. Resnick MJ, Koyama T, Fan KH et al. Long-term functional outcomes after treatment for localized prostate cancer. N Engl J Med 2013; 368: 436-445.

4. Stanford JL, Feng Z, Hamilton AS et al. Urinary and sexual function after radical prostatectomy for clinically localized prostate cancer: the Prostate Cancer Outcomes Study. JAMA 2000; 283: 354-360.

5. Zelefsky MJ, Fuks Z, Hunt M et al. High-dose intensity modulated radiation therapy for prostate cancer: early toxicity and biochemical outcome in 772 patients. Int J Radiat Oncol Biol Phys 2002; 53: 1111-1116.

6. Loeb S, Vellekoop A, Ahmed HU et al. Systematic review of complications of prostate biopsy. Eur Urol 2013; 64: 876-892.

7. Nam RK, Saskin R, Lee Y et al. Increasing hospital admission rates for urological complications after transrectal ultrasound guided prostate biopsy. J Urol 2010; 183: 963-969.
8. Davison BJ, Goldenberg SL. Patient acceptance of active surveillance as a treatment option for low-risk prostate cancer. Br J Urol 2011; 108: 1787-1793.

9. Pickles T, Ruether JD, Weir L et al. Psychosocial barriers to active surveillance for the management of early prostate cancer and a strategy for increased acceptance. Br J Int 2007; 100: 544-551.

10. Van den Bergh RC, Korfage IJ, Bangma CH. Psychological aspects of active surveillance. Curr Opin Urol 2012; 22: 237242.

11. Hamdy FC, Donovan JL, Lane JA et al. 10-Year outcomes after monitoring, surgery, or radiotherapy for localized prostate cancer. N Engl J Med 2016; 375: 1415-1424.

12. Langley S, Ahmed HU, Al-Qaisieh B et al. Report of a consensus meeting on focal low dose rate brachytherapy for prostate cancer. Br J Urol 2012; 109 (s1): 7-16.

13. Donaldson IA, Alonzi R, Barratt D et al. Focal therapy: patients, interventions, and outcomes - a report from a consensus meeting. Eur Urol 2015; 67: 771-777.

14. Bott SR, Ahmed HU, Hindley RG et al. The index lesion and focal therapy: an analysis of the pathological characteristics of prostate cancer. BJU Int 2010; 106: 1607-1611.

15. Huang CC, Deng FM, Kong MX et al. Re-evaluating the concept of "dominant/index tumor nodule" in multifocal prostate cancer. Virchows Arch 2014; 464: 589-594.

16. Noguchi M, Stamey TA, McNeal JE et al. Prognostic factors for multifocal prostate cancer in radical prostatectomy specimens: lack of significance of secondary cancers. J Urol 2003; 170: 459-463.

17. Villers A, McNeal JE, Freiha FS, Stamey TA. Multiple cancers in the prostate. Morphologic features of clinically recognized versus incidental tumors. Cancer 1992; 70: 2313-2318.

18. Wise AM, Stamey TA, McNeal JE, Clayton JL. Morphologic and clinical significance of multifocal prostate cancers in radical prostatectomy specimens. Urology 2002; 60: 264-269.

19. Prostate Imaging Reporting And Data System (PI-RADS) American College of Radiology. Available at: https://www.acr. org/Quality-Safety/Resources/PIRADS. Accessed: 1 May 2015.

20. Barentsz JO, Richenberg J, Clements R et al. ESUR prostate MR guidelines 2012. Eur Radiol 2012; 22: 746-757.

21. Kozlowski P, Chang SD, Meng R et al. Combined prostate diffusion tensor imaging and dynamic contrast enhanced MRI at $3 T$ - quantitative correlation with biopsy. Magn Res Imag 2010; 28: 621-628.

22. Bucci J, Spadinger I, Hilts M et al. Urethral and periurethral dosimetry in prostate brachytherapy: is there a convenient surrogate? Int J Radiat Oncol Biol Phys 2002; 54: 1235-1242.

23. Paterson R, Parker HM. A dosage system for interstitial radium therapy. Br J Radiol 1938; 11: 252-266.

24. Mahdavi SS, Spadinger IT, Chng NT et al. Robustness to source displacement in dual air kerma strength planning for focal low-dose-rate brachytherapy of prostate cancer. Brachytherapy 2016; 15: 642-649.

25. Chng N, Spadinger I, Morris WJ et al. Prostate brachytherapy postimplant dosimetry: Automatic plan reconstruction of stranded implants. Med Phys 2011; 38: 327-342.

26. Nir G. Prostate registration using magnetic resonance elastography for cancer localization. Doctoral dissertation, University of British Columbia, 2014.

27. Mahdavi SS, Spadinger I, Chng N et al. Semiautomatic segmentation for prostate brachytherapy: dosimetric evaluation. Brachytherapy 2013; 12: 65-76.

28. LENT SOMA scales for all anatomic sites. Int J Radiat Oncol Biol Phys 1995; 31: 1049-1091.

29. Cosset JM, Cathelineau X, Wakil G et al. Focal brachytherapy for selected low-risk prostate cancers: a pilot study. Brachytherapy 2013; 12: 331-337. 
30. Al-Qaisieh B, Mason J, Bownes P et al. Dosimetry modeling for focal low-dose-rate prostate brachytherapy. Int J Radiat Oncol Biol Phys 2015; 92: 787-793.

31. Royal Surry County Hospital NHS Foundation Trust. Hemiablative prostate brachytherapy (HAPpy). In: ClinicalTrials. gov. Bethesda (MD): National Library of Medicine (US) 2000. Availableat:https://clinicaltrials.gov/ct2/show/NCT02632669 NLM Identifier: NCT02632669. Accessed 12 February 2016.

32. University of Pittsburgh. Prospective evaluation of focal brachytherapy using Cesium-131 for patients with low risk prostate cancer. In: ClinicalTrials.gov. Bethesda (MD): National Library of Medicine (US) 2000. Available at: https:// clinicaltrials.gov/ct2/show/NCT02290366 NLM Identifier: NCT02290366. Accessed 12 February 2016.

33. St George Hospital, Australia. Hemiablative focal brachytherapy pilot study. In: ClinicalTrials.gov. Bethesda (MD): National Library of Medicine (US) 2000. Available at: https:// clinicaltrials.gov/ct2/show/NCT02643511 NLM Identifier: NCT02643511. Accessed 12 February 2016.

34. Institute Claudius Regaud, France. Phase II study of feasibility of focal therapy for prostate cancer of good prognosis with permanent I125 localized implant. In: ClinicalTrials. gov. Bethesda (MD): National Library of Medicine (US) 2000. Available at: https://clinicaltrials.gov/ct2/show/ NCT01902680 NLM Identifier: NCT01902680. Accessed 19 December 2016.

35. British Columbia Cancer Agency. Focal therapy for prostate cancer - A pilot study of focal low dose rate brachytherapy (FTPC). Bethesda (MD): National Library of Medicine (US) 2000. Available at: https://clinicaltrials.gov/ct2/show/NCT01830166 NLM Identifier: NCT01830166. Accessed 12 February 2016.

36. Kunogi H, Wakumoto Y, Yamaguchi N et al. Focal partial salvage low-dose-rate brachytherapy for local recurrent prostate cancer after permanent prostate brachytherapy with a review of the literature. J Contemp Brachytherapy 2016; 8: 167-174.

37. Kumar AM, Smith KL, Reddy CA et al. Safety and efficacy of salvage low-dose-rate brachytherapy for prostate bed recurrences following radical prostatectomy. J Contemp Brachytherapy 2015; 7: 241-246.

38. Mayes JM, Mouraviev V, Sun L et al. Can the conventional sextant prostate biopsy accurately predict unilateral prostate cancer in low-risk, localized, prostate cancer? Urol Oncol 2011; 29: 166-170.

39. Washington SL, Bonham M, Whitson JM et al. Transrectal ultrasonography-guided biopsy does not reliably identify dominant cancer location in men with low-risk prostate cancer. Br J Urol 2012; 110: 50-55

40. Mouraviev V. Histological Trends and the Index Lesion in Localized Prostate Cancer. Focal Therapy in Prostate Cancer 2011; 19: 17.

41. Barzell WE, Melamed MR. Appropriate patient selection in the focal treatment of prostate cancer: the role of transperineal 3-dimensional pathologic mapping of the prostate a 4-year experience. Urology 2007; 70: S27-35.

42. Merrick GS, Taubenslag W, Andreini H et al. The morbidity of transperineal template-guided prostate mapping biopsy. Br J Urol 2008; 101: 1524-1529.

43. Losa A, Gadda GM, Lazzeri M et al. Complications and quality of life after template-assisted transperineal prostate biopsy in patients eligible for focal therapy. Urology 2013; 81: 1291-1296.

44. Kepner GR, Kepner JV. Transperineal prostate biopsy: analysis of a uniform core sampling pattern that yields data on tumor volume limits in negative biopsies. Theor Biol Med Model 2010; 7: 23.

45. Stamey TA, Freiha FS, McNeal JE et al. Localized prostate cancer. Relationship of tumor volume to clinical significance for treatment of prostate cancer. Cancer 1993; 71(S3): 933-938.
46. Ouzzane A, Puech P, Lemaitre L et al. Combined multiparametric MRI and targeted biopsies improve anterior prostate cancer detection, staging, and grading. Urology 2011; 78: 13561362.

47. De Rooij M, Hamoen EH, Fütterer JJ et al. Accuracy of multiparametric MRI for prostate cancer detection: a meta-analysis. AJR Am J Roentgenol 2014; 202: 343-351.

48. Delongchamps NB, Rouanne M, Flam T et al. Multiparametric magnetic resonance imaging for the detection and localization of prostate cancer: combination of T2-weighted, dynamic contrast-enhanced and diffusion-weighted imaging. Br J Int 2011; 107: 1411-1418.

49. Turkbey B, Mani H, Shah V et al. Multiparametric 3T prostate magnetic resonance imaging to detect cancer: histopathological correlation using prostatectomy specimens processed in customized magnetic resonance imaging based molds. J Urol 2011; 186: 1818-1824.

50. Schoots IG, Petrides N, Giganti F et al. Magnetic resonance imaging in active surveillance of prostate cancer: a systematic review. Eur Urol 2015; 67: 627-636.

51. Sahebjavaher RS, Nir G, Gagnon LO et al. MR elastography and diffusion-weighted imaging of ex vivo prostate cancer: quantitative comparison to histopathology. NMR in Biomedicine 2015; 28: 89-100.

52. Honarvar M, Sahebjavaher R, Sinkus R et al. Curl-based finite element reconstruction of the shear modulus without assuming local homogeneity: time harmonic case. IEEE Trans Med Imaging 2013; 32: 2189-2199.

53. Mahdavi SS, Moradi M, Wen X et al. Evaluation of visualization of the prostate gland in vibro-elastography images. Med Image Anal 2011; 15: 589-600.

54. Moradi M, Mahdavi SS, Nir G et al. Ultrasound RF time series for tissue typing: first in vivo clinical results. In SPIE Medical Imaging 2013; 86701I-86701I-8.

55. Turgay E, Salcudean S, Rohling R. Identifying the mechanical properties of tissue by ultrasound strain imaging. Ultrasound Med Biol 2006; 32: 221-235. 